\title{
Evaluation of Echinacea for Treatment of the Common Cold
}

\author{
Joel T. Giles, Pharm.D., Cuthbert T. Palat, III, Pharm.D., Susan H. Chien, Pharm.D., \\ Ziba Gorji Chang, Pharm.D., and Daniel T. Kennedy, Pharm.D.
}

Considered to have immunostimulating activity, echinacea is a widely used phytomedicinal for treatment of the common cold and upper respiratory tract infections (URTIS). We reviewed the literature from the MEDLINE database (January 1966-July 1999), International Pharmaceutical Abstracts (IPA) online database, Cambridge Scientific Abstracts Biological Sciences online database, Alt-Health Watch online database, EMBase CD-ROM database, and references from published articles, reviews, and letters to evaluate evidence from clinical trials of echinacea's purported efficacy for treating or preventing URTIs. Twelve clinical studies published from 1961-1997 concluded that echinacea was efficacious for treating the common cold, but the results are unclear due to inherent flaws in study design. Five trials were published since 1997; two showed that echinacea lacked efficacy for treating and preventing URTI symptoms, and three concluded that it was effective in reducing the frequency, duration, and severity of common cold symptoms. Again, these results are unclear because of methodologic uncertainties, such as small populations and use of noncommercially available, nonstandardized dosage forms. Although evidence for echinacea's efficacy is inconclusive, it appears to be safe. Patients without contraindications to it may not be dissuaded from using an appropriate preparation to treat the common cold.

(Pharmacotherapy 2000;20(6):690-697)

\section{OUTLINE}

Trends in Use of Herbal Agents

Background of Echinacea

Clinical Trials of Echinacea

Conclusion

No acute illness is more prevalent in the United States than the common cold. ${ }^{1}$ Adults typically suffer 2-4 colds/year and children may experience as many as 10 colds/year. The economic impact is substantial, with an

From the School of Pharmacy, Virginia Commonwealth University, Medical College of Virginia Campus (Drs. Giles and Kennedy), CVS Pharmacy (Dr. Palat), and Target Pharmacy (Dr. Chang), Richmond, Virginia; and CVS Pharmacy, Martinsville, Virginia (Dr. Chien).

Address reprint requests to Daniel T. Kennedy, Pharm.D., Virginia Commonwealth University, Medical College of Virginia Campus, P.O. Box 980533, Richmond, VA 232980533. estimated $\$ 1.5$ billion spent annually on caring for these patients in physicians' offices and nearly $\$ 2$ billion on nonprescription cough and cold treatments. ${ }^{1,2}$ Forty percent of time lost from jobs and $30 \%$ of absences from school can be attributed to colds. ${ }^{1}$

Various organisms cause the cold. The singlestranded RNA rhinovirus is the most common, causing 10-40\%. ${ }^{1}$ Other viruses are coronaviruses, parainfluenza viruses, adenoviruses, echoviruses, and coxsackieviruses. Colds due to rhinovirus are most prevalent in early fall and late spring, and coronavirus strains gain prevalence during winter months in the United States. ${ }^{3,4}$ Viral transmission most frequently occurs from the hands of donors directly or indirectly to the hands of recipients who touch their eyes or nose, the preferred viral entry sites. ${ }^{3}$ Infection begins in the posterior adenoidal region of the 
nasopharynx, which contains receptor targets for the rhinovirus. ${ }^{5}$ Viral replication peaks in 48 hours and declines, but viral shedding can continue for up to 3 weeks. ${ }^{5}$

With infection, an inflammatory response follows that causes characteristic nasal symptoms (discharge, sneezing, stuffiness), pharyngeal symptoms (sore, scratchy throat), lower respiratory symptoms (cough, hoarseness), and constitutional symptoms (headache, fever, myalgia). ${ }^{3}$ Currently available treatments for the common cold are limited to alleviation of symptoms. Nasal symptoms can be reduced by first-generation antihistamines and oral or topical $\alpha$-adrenergic agonists. ${ }^{6}$ The most common side effects of these agents are drowsiness, nasal dryness, and rebound congestion (rhinitis medicamentosa), respectively. Analgesics are indicated for myalgia and headache. Cough suppressants, such as codeine and dextromethorphan, and expectorants, such as guaifenesin, are typical components of anticold products, but have no efficacy in the common cold. ${ }^{2}$

\section{Trends in Use of Herbal Agents}

The past decade has seen a dramatic rise in the popularity of alternative medicine. According to a national survey, use of these therapies in 1997 ranged from $32-54 \%$ in the demographic groups examined. ${ }^{7}$ Millions of Americans now rely on alternative products for a variety of ailments. The use of herbs by American adults increased nearly $70 \%$ in 1998, with three-fifths of consumers taking two or more products on a daily basis. ${ }^{8}$ Another study reported that 34\% of 1539 adults used at least one unconventional therapy over the previous year. ${ }^{9}$ The number of people in the United States who used alternative therapies increased from 60 million in 1990 to 83 million in $1997 .^{7}$

For acute, self-limiting conditions such as colds, people may prefer herbal remedies because professional care is inconvenient, not readily available, or too costly. ${ }^{10}$ Conventional treatment offers only symptomatic relief, so cold sufferers may seek alternatives for potentially improved responses. Echinacea, one of the top three selling herbal medicines in the United States, ${ }^{8}$ has been evaluated in clinical trials for treatment and prevention of the common cold.

\section{Background of Echinacea}

Echinacea played an important role in Native
American medicine. It is a perennial member of the daisy family, native to Midwestern North America. Common names are American coneflower, black Susan, purple coneflower, and narrow-leafed coneflower. Echinacea extracts were commonly prescribed until the introduction of sulfa drugs in the 1930s. The herb regained popularity in the United States in the 1980s.

Echinacea purpurea dietary supplements typically consist of fresh, above-ground portions of the plant harvested at flowering time, although the entire plant has been used for medicinal purposes. Of the nine species, E. angustifolia, E. purpurea, and E. pallida are the most commonly used to treat the common cold and upper respiratory tract infections (URTIS) Although $E$. angustifolia has long been considered to have the greatest immunostimulating activity, this was recently disputed.11-13 As E. purpurea is the easiest to grow commercially, it may become the most used species in the United States.

Purported uses of echinacea are supportive therapy for colds and chronic infections of the respiratory tract, treatment of lower urinary tract infections, and treatment of superficial wounds when applied externally. In human and animal experiments, preparations given orally or parenterally produced immunostimulating effects. Among other physiologic actions, the number of white blood cells is increased, phagocytosis by human granulocytes is activated, and body temperature is elevated. ${ }^{14}$ Other actions are antiviral, antiinflammatory, and antibacterial properties, which were consistently reported from in vitro experiments.

Echinacea formulations may contain any or all of the principal three species in the form of its herb (aerial portion) and/or root. Most work investigating echinacea has been performed in Germany with a proprietary formulation of $E$. purpurea juice (Echinacin). This form is not currently available in the United States. The Commission E monographs represent the official position of the German government on different herbal therapies based on scientific data. They recommend the root of $E$. pallida orally and the herb of $E$. purpurea orally and parenterally. ${ }^{15}$ The root of $E$. purpurea, the herb of $E$. pallida, and any part of $E$. angustifolia are not recommended due to lack of data.

\section{Clinical Trials of Echinacea}

The validity of controlled trials examining the efficacy of echinacea as an immunomodulator 
was previously reviewed. ${ }^{16}$ An extensive literature search yielded 26 controlled trials published from 1961-1992, of which 6 studied echinacea for treatment and 6 for prophylaxis of URTIS. ${ }^{17-28}$ The primary authors of 11 of these studies concluded that echinacea was superior to control treatment. Based on a systematic assessment of methodologic quality to estimate evidence supporting these conclusions, however, echinacea's efficacy for treating or preventing URTIs or the common cold was unclear in all but 1 of the 12 studies. That study administered 900 $\mathrm{mg} /$ day of an ethanolic extract of $E$. purpurea root, which was thought to have verified efficacy for relieving URTI symptoms according to the study's design and analysis. ${ }^{17}$ Currently, the German Commission $\mathrm{E}$ does not recommend use of the root of $E$. purpurea. Other studies contained serious methodologic flaws and had insufficient or flawed statistical analyses. Many lacked defined URTI diagnostic criteria, adequate sample sizes, randomization, description of the randomization process, analysis of baseline subject comparability, descriptions of treatment interventions, defined outcome criteria, patient blinding, evaluator blinding, and numbers of and reasons for subject withdrawal. The reviewers concluded that some evidence exists to support the proposed benefits of echinacea for treating and preventing URTIs. However, evidence from methodologically inadequate and heterogeneous clinical trials is insufficient for confident dosage or formulation recommendations.

A second review was conducted of 13 randomized, double-blind, placebo-controlled trials of echinacea for prevention or treatment of URTIS. ${ }^{29}$ Eight of the nine treatment trials reported benefit against URTI symptoms. The four trials of URTI prevention resulted in statistically insignificant evidence for prophylactic efficacy. Despite the trials' methodologic deficiencies, the reviewers concluded that current evidence suggests a role for echinacea in the treatment, but not prophylaxis, of acute URTIs.

Since the former review, ${ }^{16}$ a number of clinical trials examined echinacea for treatment and prevention of the common cold. A three-arm (two echinacea preparations and placebo), randomized, double-blind trial conducted at four military installations and a single industrial plant studied root extracts for prevention of URTIs. ${ }^{30}$ Subjects were included if they were 18-65 years of age and were not suffering from an acute illness. Subjects were excluded if they had acute respiratory tract infection in the last 7 days; serious progressive disease such as HIV/AIDS; therapy within the past 2 weeks with systemic corticosteroids, antibiotics, or immunostimulants; allergy to the Compositae family, which contains the genus Echinacea; or pregnancy. Of 302 subjects, 289 were evaluated in the intent-to-treat analysis. Two ethanolic extracts (1:11 plant extract ratio in $30 \%$ alcohol from the roots of $E$. angustifolia or E. purpurea) or placebo were given orally for 12 weeks. Subjects were instructed to take 50 drops $(91 \mathrm{mg})$ of the assigned preparation twice/day Monday through Friday.

Time to first URTI was the primary outcome measure. Secondary outcome measures were the number of subjects with at least one infection, adverse effects, and quality of life assessments. Subjects completed a quality of life questionnaire and an activities of daily living questionnaire at the beginning of the study and a quality of life questionnaire at study conclusion. Results of the main outcome measure of mean time to first URTI was 66 days in the E. angustifolia arm, 69 days in the $E$. purpurea arm, and 65 days in the placebo arm $(p=0.49)$. The relative risk compared with placebo for having at least one URTI was 0.87 (95\% confidence interval [ $\mathrm{CI}$ ] $0.59-1.30$ ) for subjects receiving $E$. angustifolia and 0.80 (95\% $\mathrm{Cl} 0.53-1.31)$ for those receiving E. purpurea. Furthermore, no significant differences between treatment and placebo groups were found regarding number, severity, or duration of URTI symptoms, quality of life measures, and adverse effects. The preparations appeared to be well tolerated, as the most frequent adverse effects reported were minor gastrointestinal symptoms and headachedizziness, probably due to the ethanol component of the extract. These adverse effects occurred at a rate of less than $10 \%$ in each treatment group and were not more prevalent with echinacea than with placebo. The authors concluded that no prophylactic effect was shown, but speculated that a $10-20 \%$ relative risk reduction in time to first URTI may exist with echinacea. It was hypothesized that larger trials with more than 1000 participants would be necessary to provide the statistical power to see significant preventive benefits.

A limitation of the study was use of roots of $E$. purpurea and E. angustifolia. The German Commission $\mathrm{E}$ recommends only the root form of E. pallida, not the other two species. Furthermore, use of oral echinacea preparations beyond 8 consecutive weeks, unlike the 12 weeks 
in this study, is not recommended. ${ }^{15}$ Symptoms of URTI may have been alleviated by other agents or treatments, as subjects were not required to report such therapy while receiving study preparations. $^{31}$ Per protocol, participants did not take the assigned treatment daily, skipping Saturday and Sunday each week. Also, it is difficult to assess the appropriateness of the dosages of echinacea because they were not standardized.

A randomized, double-blind, placebocontrolled trial assessed Echinagard, a proprietary preparation of the herb of $E$. purpurea, in patients with acute URTI. ${ }^{32}$ One hundred twenty subjects enrolled, 60 in the treatment group and 60 in the placebo arm. Demographics were similar for the two groups. Inclusion criteria were a history of at least three URTIs within the past 6 months and the presence of initial signs of an URTI. Exclusion criteria were URTI within a week of starting the study, pregnancy or lactation, immunologic disease or current immunotherapy, and history of hypersensitivity to plants of the daisy family (Asteraceae) containing the genus Echinacea.

Subjects in the treatment group drank 4 ounces of water with 20 drops of $E$. purpurea solution every 2 hours on day 1 , and the same dose 3 times/day on days 2-10. They used a daily diary to record symptoms. They also completed a questionnaire regarding the extent and duration of symptoms and use of treatment preparations on day 10. The primary outcome measure was the time until symptoms improved and treatment ended. Only $40 \%$ of the echinacea group developed a "real" cold (defined as fully expressed symptoms of a URTI) compared with $60 \%$ in the placebo group $(p=0.044)$. Because some subjects did not develop a real cold, a subgroup analysis of those who did was performed and showed that time to symptom improvement was shorter in the treatment group versus the placebo group ( 4 vs 8 days, respectively). The authors concluded that Echinagard begun at the first sign of a URTI inhibited progression and resulted in quicker relief of symptoms than placebo.

The study's results are limited by the methodology. Patients' symptoms were subjectively self-reported in a diary that was not explained by the authors or proven to be a valid instrument. Also, whether or not a real cold had developed was determined by a physician after the 10-day treatment, at which time symptoms had already resolved. No statistical analysis was presented for the subgroup analysis of patients who developed a real cold. The mean dose of echinacea administered on the first day is unclear. The methodology does not distinguish if subjects received echinacea every 2 hours around the clock for 12 doses or if they took doses only while awake. It should be noted that the results support only use of Echinagard and not other echinacea preparations. This product is marketed in the United States as EchinaGuard and EchinaGuard Pro. ${ }^{33}$

The efficacy of $E$. pallida root versus placebo was evaluated in patients with signs and symptoms of a URTI of less than 3 days' duration. ${ }^{34}$ Patients were included in this double-blind, placebo-controlled trial based on a score of 15 or higher on a questionnaire of URTI symptoms. Weakness, sweating, tearing, burning eyes, sore throat, earache, pain in arms and legs, and headache were scored on a scale of $0-3$ (none-severe). Exclusion criteria included autoimmune disorders, other infections, and therapy with drugs that might interact with the herbal preparation. The authors considered an elevated differential neutrophil count as evidence of bacterial infection and elevated differential Iymphocyte count as evidence of viral infection. One hundred sixty subjects, evenly divided between treatments, received $900 \mathrm{mg}$ of liquid $\mathrm{E}$. pallida root extract/day or an indistinguishable placebo for 8-10 days. Outcomes were resolution of symptoms and duration of illness. Treatment with $\mathrm{E}$. pallida reduced the duration of illness from 13 to 9.8 days for bacterial infections and from 13 to 9.1 days for viral infections. The decrease was significant ( $p<0.001$ ) between the intervention and placebo groups.

Symptoms were assessed by a physician at baseline and 3-4 and 8-10 days after study entry. Subjects were included if they experienced symptoms for up to 3 days. The authors do not present a mean duration of symptoms before receiving placebo or treatment, and it is unknown when subjects began either arm. No comparison was made between subjects who began treatment on day 1 or less versus those who started on day 3 of symptom onset. Perhaps those who started therapy earlier may have had a significant decrease in the duration of illness compared with those beginning treatment later in the course of illness. Although the duration of illness was shorter in the treatment arm, subjects still experienced symptoms for 9-10 days. Clinicians should consider if 8-10 days of $E$. pallida is worth while, as patients continued to 
perceive symptoms for up to 10 days, only 3 days less than those who were not treated. The authors did note that symptom severity declined faster in the treatment group.

A randomized, double-blind, placebocontrolled trial was conducted to determine the effects of $E$. purpurea extract on the frequency, duration, and severity of URTIs. ${ }^{35}$ Subjects were treated with either $4 \mathrm{ml}$ of echinacea extract or placebo juice twice/day for 8 weeks. Eligible participants had a history of frequent colds, with documentation of three or more respiratory tract infections or colds within the previous year. Exclusion criteria were age under 12 years, an acute infection within 1 week of recruitment, pregnancy or lactation, treatment with immunostimulating agents, allergy to echinacea, severe underlying disease or immunosuppression, inability to give informed consent, and lack of reliability for follow-up. Of 109 enrolled, 108 completed the study. Active treatment consisted of the expressed juice of the whole flowering $E$. purpurea plant, without the roots, in $22 \%$ alcohol. Placebo and extract were provided by Madus AG, Cologne, Germany, and were reported to be indistinguishable in appearance, color, and flavor. A medical history, physical examination, and hematologic tests were performed at baseline, week 4 , and week 8 , or at any time a subject experienced signs and symptoms of a URTI.

Primary outcome measures were frequency and severity of URTIS and colds during the 8-week study. Sixty-five percent of patients in the treatment group and $70 \%$ in the placebo group experienced one or more infections (mean 0.78 and 0.93 , respectively, $p=0.33$ ). The median duration of colds and URTIs was 4.5 days and 6.5 days, respectively $(p=0.45)$. Signs and symptoms of a cold were classified as mild, moderate, or severe based on predetermined criteria. No significant differences were found for the severity of colds and number of infections per severity category. The E. purpurea juice did not significantly reduce the frequency, duration, or severity of URTIs. Common adverse effects were central nervous system symptoms (somnolence, dizziness, headache) and gastrointestinal symptoms (nausea, mild epigastric pain, constipation), each occurring in approximately $7 \%$ of patients receiving echinacea, not significantly more frequent than in those receiving placebo.

The population was small, 54 subjects in each group, and was not powerful enough to detect smaller differences between groups. Like other studies that used an echinacea extract with a high ethanol content, it is unclear what effect if any the alcohol may have on cold symptoms; it is likely that it did contribute to the frequency of adverse central nervous system and gastrointestinal symptoms. The results may have been somewhat confounded as $13 \%$ of subjects in the echinacea group had received influenza vaccinations before study entry versus only $4 \%$ of placebo subjects; however, this difference was not statistically significant $(p=0.16)$. Again, the dosage form was not standardized, which creates obscurity when comparing studies and when assessing the appropriateness of the regimens.

A randomized, double-blind, placebocontrolled trial examined which preparation and dosage is most appropriate for treatment of the common cold. ${ }^{36}$ Otherwise healthy 18 -year-olds susceptible to colds were assigned to one of four treatments. One group received the trade drug Echinaforce, which contained $6.78 \mathrm{mg}$ of $E$. purpurea extract of $95 \%$ herb and $5 \%$ root. Two groups received fresh plant extracts, $48.27 \mathrm{mg}$ of a concentrated $95 \%$ herb and $5 \%$ root of $E$. purpurea or $29.60 \mathrm{mg}$ of $E$. purpurea root only. The fourth group was assigned to placebo. At the first sign or symptom of a cold, patients began taking two tablets of the study agent 3 times/day and were instructed to see physician investigators for assessment on day 1 or 2 of the cold. Treatment continued until symptoms resolved or for a maximum of 7 days. At that point, subjects returned to the physician for a final assessment. Of 559 subjects initially recruited, 246 developed cold symptoms and returned for assessment to be included in the intent-to-treat analysis. Sixty-five of the 246 patients were excluded from perprotocol analysis due to protocol violations or use of other drugs.

A subjective system of scoring 12 common cold systems ( no symptoms $=0$; mild, moderate, or severe symptoms $=1,2$, or 3, respectively) was employed. The sum of values assigned to each symptom yielded a complaint index score. According to the the per-protocol analysis, Echinaforce and the concentrated E. purpurea preparation (48.27 mg/tablet) significantly reduced the mean physician-assessed complaint index from day 1 or 2 of symptoms (mean $\pm 95 \%$ $\mathrm{Cl} 8.8 \pm 1.1$ and $8.0 \pm 1.1$, respectively) to the final visit ( $3.5 \pm 1.2$ and $2.6 \pm 0.9$, respectively), for relative mean reductions of $62.7 \pm 11 \%$ and $64.3 \pm 12 \%$, respectively. The final symptom resolution was better than placebo $(29.3 \pm 18 \%$, $p=0.020$ for Echinaforce and $p=0.003$ for the 
concentrated preparation). The other plant preparation of $\mathrm{E}$. purpurea root (29.60 $\mathrm{mg} / \mathrm{tablet})$ did not show a statistically significant mean relative reduction in complaint index scores $(44.8 \pm 20 \%, p=0.060)$ versus placebo. These results were similar to those for the intent-totreat group. Mean relative reductions $\pm 95 \% \mathrm{Cl}$ for Echinaforce, concentrated extract, and rootonly extract versus placebo $(33.6 \pm 17 \%)$ were $58.7 \pm 10 \%(p=0.045), 58.1 \pm 11 \%(p=0.027)$ ， and $46.1 \pm 16 \%(p=0.133)$, respectively.

Secondary outcomes examined patient diaries for scoring symptoms, physician and patient judgment of treatment efficacy, and adverse effects. Per-protocol analysis showed a mean relative reduction in complaint index significantly better than placebo $(37.0 \pm 13 \%)$ for Echinaforce and concentrated extract (50.6 \pm $11 \%, p=0.032 ; 55.9 \pm 9 \%, p=0.010$, respectively). The root extract was not better than placebo in reducing the mean relative reduction in complaint index scores (35.2 $\pm 17 \%, p=0.271$ ). Both physicians and patients thought that Echinaforce and concentrated extract were more often effective than not in reducing cold symptoms. The most common adverse effects were mild gastrointestinal symptoms, which occurred infrequently and not more often in the treatment than in the placebo group.

The authors concluded that Echinaforce and the concentrated E. purpurea extract, of similar composition to Echinaforce at a 7-fold higher dosage, effectively reduced cold symptoms better than placebo or the E. purpurea root extract. Also, the lower-dose Echinaforce tablets achieved nearly the same symptom reduction as the high dose of concentrated herb and root extracts. The study analyzed final symptom scores only on resolution of symptoms or after 7 days of treatment. No data were provided on the duration of symptom resolution, only that the complaint index scores decreased in 7 days or fewer. A more appropriate conclusion would be that Echinaforce and the concentrated extract reduced cold symptoms better than placebo in 7 days or fewer. Complaint index scores at day 1 or 2 of the cold are interesting as the maximum score for the most severe cold is 36 , and mean scores ranged from 7.7-10.9 in the four groups reported from patient diaries and physicians. One can question if the colds were less severe than they would have been in a larger population, or that if complaint index is an appropriate instrument to assess cold severity. $\mathrm{No}$ validation of the subjectively determined complaint index is provided. Also, on final examination at 7 days or when the subject's symptoms had resolved to a complaint index score of 2 or less, patients receiving Echinaforce and concentrated extract were still scoring symptoms from a mean range of 2.6-5.2. This means that even though the reduction in symptoms in 7 days was better with active treatment than with placebo, patients were experiencing symptoms even after 7 days of taking the preparations.

Table 1 summarizes the results of the aforementioned clinical trials evaluating echinacea.

\section{Conclusion}

Echinacea appears to be well tolerated with a low frequency of adverse effects, such as mild dyspepsia, headache, and dizziness. Serious allergic reactions involving anaphylaxis are rare, but caution is advised for patients with a history of hypersensitivity to the daisy family (sunflower seeds, ragweed). With minimal toxicity and favorable side effect profile, patients may not be dissuaded from taking echinacea unless they have an autoimmune disease, human immunodeficiency viral infection, multiple sclerosis, tuberculosis, pregnancy, or lactation, or are receiving immunosuppressive agents. ${ }^{37}$ Echinacea preparations have not been evaluated in children and infants, and are not recommended for them. It is difficult to offer dosing recommendations as preparations are not standardized to an active constituent. Some preparations use echinacoside as a standard; it is thought that this constituent is not active, and it is not even found in $E$. purpurea. ${ }^{38}$ A reasonable recommended dosage is 2-3 $\mathrm{ml}$ of $E$. purpurea juice or $1-2 \mathrm{ml}$ of an extract taken 3-4 times/day in juice or water or sublingually. ${ }^{39}$ One hundred fifty milligrams to $300 \mathrm{mg}$ of a $6.5: 1$ dry powdered E. purpurea extract can be taken in a solid oral dosage form 3 times/day if the juice or fluid extract cannot be tolerated. ${ }^{37,39}$ A tincture of 1:5 (45\% ethanol) 1-5 $\mathrm{ml} 3$ times/day is recommended by the British Herbal Pharmacopoeia. ${ }^{40}$ Echinacea's immunostimulating properties may decline with continued use, so preparations should not be taken for longer than 8 consecutive weeks. ${ }^{15,} 37,39$ After a drug holiday, the herb may be restarted for another 8 weeks. ${ }^{41}$

Studies evaluated here support echinacea in the treatment of a URTI, but not to prevent infection. Although the effects are not dramatic, they may 
Table 1. Echinacea Trials Since $1994^{16}$

\begin{tabular}{|c|c|c|c|c|c|}
\hline $\begin{array}{l}\text { Study } \\
\text { Design }\end{array}$ & $\begin{array}{l}\text { No. of } \\
\text { Pts. }\end{array}$ & Echinacea Formulation & Dosage & Duration & Results \\
\hline \multirow[t]{2}{*}{$\begin{array}{l}\mathrm{R}, \mathrm{DB} \\
\mathrm{PC}^{30}\end{array}$} & 289 & $\begin{array}{l}\text { E. angustifolia 1:11 root extract } \\
\text { in } 30 \% \text { EtOH }\end{array}$ & $\begin{array}{l}50 \mathrm{gtt} \text { b.i.d. M-F } \\
\text { (91 mg echinacea) }\end{array}$ & 12 wks & $\begin{array}{l}\text { No significant difference in mean time } \\
\text { until first URTI between treatment } \\
\text { groups. }\end{array}$ \\
\hline & & $\begin{array}{l}\text { E. purpurea 1:11 root extract } \\
\text { in } 30 \% \mathrm{EtOH}\end{array}$ & $\begin{array}{l}50 \mathrm{gtt} \text { b.i.d. M-F } \\
\text { (91 mg echinacea) }\end{array}$ & 12 wks & $\begin{array}{l}\text { E. angustifolia } 66 \text { days, E. purpurea } 69 \\
\text { days, placebo } 65 \text { days }(p=0.49) \text {. } \\
\text { No significant differences between } \\
\text { treatment and placebo regarding relative } \\
\text { risk for one URTI or number, duration, } \\
\text { or severity of symptoms. }\end{array}$ \\
\hline $\begin{array}{l}\mathrm{R}, \mathrm{DB} \\
\mathrm{PC}^{32}\end{array}$ & 120 & $\begin{array}{l}\text { E. purpurea herb squeezed sap } \\
\text { in } 4 \text { oz water }\end{array}$ & $\begin{array}{l}20 \text { gtt q } 2 \text { hrs on } \\
\text { day } 1 \text { of URTI, } \\
\text { then } 20 \text { gtt t.i.d. } \\
\text { x } 10 \text { days }\end{array}$ & $\begin{array}{l}10 \text { days } \\
\text { or until } \\
\text { symptom } \\
\text { resolution }\end{array}$ & $\begin{array}{l}60 \% \text { of treated patients did not develop } \\
\text { fully expressed URTI symptoms } \\
\text { ( } p=0.044) \text {. } \\
\text { Symptoms improved in } 4 \text { days for treated } \\
\text { patients who developed fully expressed } \\
\text { URTI symptoms vs } 8 \text { days for placebo. }\end{array}$ \\
\hline $\begin{array}{l}\mathrm{R}, \mathrm{DB} \\
\mathrm{PC}^{34}\end{array}$ & 160 & E. pallida root extract & 900 mg/day & 8-10 days & $\begin{array}{l}\text { Significant decrease in duration of } \\
\text { bacterial infections ( } 9.8 \text { days) and viral } \\
\text { infections ( } 9.1 \text { days) vs placebo } \\
\text { ( } 13 \text { days; } p<0.001 \text { ). }\end{array}$ \\
\hline $\begin{array}{l}\mathrm{R}, \mathrm{DB}, \\
\mathrm{PC}^{35}\end{array}$ & 108 & $\begin{array}{l}\text { E. purpurea expressed juice } \\
\text { extract of whole flowering } \\
\text { aerial plant portion in } 22 \% \\
\text { EtOH }\end{array}$ & $4 \mathrm{ml}$ b.i.d. & 8 wks & $\begin{array}{l}\text { M ean no. URTIs } 0.78 / \text { patient in the } \\
\text { treatment group and } 0.93 / \text { patient } \\
\text { receiving placebo ( } p=0.33) \text {. } \\
\text { M edian duration of symptoms } 4.5 \text { days } \\
\text { with treatment and } 6.5 \text { days with placebo } \\
\text { ( } p=0.45) \text {. } \\
\text { No significant difference in severity of } \\
\text { symptoms between treatment and } \\
\text { placebo. }\end{array}$ \\
\hline $\begin{array}{l}\mathrm{R}, \mathrm{DB} \\
\mathrm{PC}^{36}\end{array}$ & $181^{a}$ & $\begin{array}{l}6.78 \mathrm{mg} \text { E. purpurea extract } \\
\text { of } 95 \% \text { herb, } 5 \% \text { root, } \\
48.27 \mathrm{mg} \text { E. purpurea } \\
\text { concentrated fresh plant } \\
\text { extract of } 95 \% \text { herb, } 5 \% \text { root, } \\
29.60 \mathrm{mg} \text { E. purpurea root extra }\end{array}$ & $\begin{array}{l}\text { At first URTI } \\
\text { symptom, } \\
2 \text { tablets t.i.d. } \\
\end{array}$ & $\begin{array}{l}7 \text { days } \\
\text { maximum }\end{array}$ & $\begin{array}{l}\text { Decrease in URTI complaint index scores } \\
\text { from day } 1 \text { or } 2 \text { of symptoms to } \\
\text { resolution for } 6.78 \text {-mg tablet and } \\
48.27 \text {-mg plant extract vs placebo } \\
\text { ( } p=0.020 \text { and } p=0.003 \text {, respectively). }\end{array}$ \\
\hline
\end{tabular}

be significant in some cases. The herb may be taken to decrease the time to resolution of signs and symptoms of a URTI early after symptom start; however, other factors must be considered. Trial results of one Echinacea species, extraction method, and dosage form cannot be extrapolated to a dissimilar preparation because chemical constituents may differ. From studies in the English language, it is uncertain which part of the plant (root, aerial portion), of which species (E. purpurea, E. angustifolia, E. pallida), in which formulation (squeezed sap, hydroalcoholic extract, dried powder extract, etc.) offers the most evidence for efficacy.

The efficacy of echinacea for treating common cold symptoms remains unclear, yet it appears to be a safe alternative for suitable patients. Trials with statistical power sufficient to detect significant differences, that are free of confounding design flaws, and that use standardized echinacea dosage forms commercially available in the United States should be conducted.

\section{References}

1. Kirkpatrick GL. The common cold. Prim Care 1996; 23(4):657-75.

2. Turner RB. Epidemiology, pathogenesis, and treatment of the common cold. Ann Allergy Asthma Immunol 1997;78(6): 531-9.

3. Lorber B. The common cold. J Gen Intern Med 1996;11(4): 229-36.

4. Hilding DA. Literature review: the common cold. Ear Nose Throat J 1994;73(9):639-43, 646-7.

5. Gwaltney JM Jr. Rhinovirus infection of the normal human airway. Am J Respir Crit Care Med 1995;152(4 pt 2):S36-9.

6. Mossad SB. Treatment of the common cold. BMJ 
1998;317(7150):33-6

7. Eisenberg DM, Davis RB, Ettner SL, et al. Trends in alternative medicine use in the United States, 1990-1997: results of a follow-up national survey. JAMA 1998;280(18):1569-75

8. Anonymous. America the beautiful (and herbal). Retail Pharmacy N ews 1998:16.

9. Eisenberg DM, Kessler RC, Foster C, Norlock FE, Calkins DR, Delbanco TL. Unconventional medicine in the United States. Prevalence, costs, and patterns of use. N Engl J Med 1993:328(4):246-52.

10. Winslow LC, Kroll DJ. Herbs as medicine. Arch Intern Med 1998;158:2192-9.

11. Murray MT. Echinacea: pharmacology and clinical applications. Am J N at M ed 1995;2:18-24.

12. Bauer R, Jurcic K, Puhlmann J, Wagner $\mathbf{H}$. Immunological in vivo and in vitro examinations of echinacea extracts. Arzneimittelforschung 1988;38(2):276-81.

13. Schumacher A, Friedberg KD. Analysis of the effect of Echinacea angustifolia on unspecified immunity of the mouse. Arzneimittelforschung 1991;41:141-7.

14. Blumenthal M, Riggins C. Popular herbs in the U.S. market: therapeutic monographs. Austin, TX: American Botanical Council 1997:1-68.

15. Blumenthal $\mathbf{M}$. The complete German Commission $\mathrm{E}$ monographs. Therapeutic guide to herbal medicines. Boston: American Botanical Council with Interpretive Medicine Communications, 1998

16. Melchart D, Linde K, Worku F, Bauer R, Wagner $\mathbf{H}$. Immunomodulation with echinacea-a systematic review of controlled clinical trials. Phytomedicine 1994;1:245-54.

17. Braunig B, Dorn M, Knick E. Echinacea purpureae radix for the enhancement of the body's own immune defense mechanisms in influenza-like infections. Z Phytother 1992;13:7-13

18. Braunig B, Knick E. Therapeutical experiences with Echinacea pallida for influenza-like infections. Naturheilpraxis 1993;1:72-5.

19. Dorn M. Treatment of influenza-like syndromes with a phytotherapeutic immunostimulatory preparation. Nat GanzheitsM ed 1990;2:314-19.

20. Reitz HD. Immunomodulation with phytotherapeutic agents: a scientific study on the example of esberitox. Notabene Medici 1990;20:362-6.

21. Vorberg G. Stimulation of the immune defense in common colds. Arztliche Praxis 1984;36:97-8.

22. Vorberg G, Schneider B. Phytotherapeutic immunostimulator decreases the duration of influenza-like syndromes. Doubleblind trial proves the enhancement of unspecific immune defense. Arztliche Forschung 1989;36:3-8.

23. Forth $\mathbf{H}$, Beuscher $\mathbf{N}$. Influence of esberitox on the frequency of the common cold. Z Allgemeinmed 1981;57:2272-5.
24. Freyer HU. Frequency of the common cold in children and possibilities for prophylaxis. Fortschr Med 1974;92:165-8.

25. Helbig G. Unspecific stimulation therapy for the prophylaxis of infections. Med Klin 1961;56:1512-14

26. Kleinschmidt $\mathbf{H}$. Trials to decrease the frequency of infections in infants with esberitox. Ther Gegenwart 1965;104:1258-62.

27. Schmidt U, Albrecht $\mathbf{M}$, Schenk N. Immunostimulator decreases the frequency of influenza-like syndromes. Doubleblind, placebo-controlled trial on 646 students of the University of Cologne. Nat GanzheitsM ed 1990;3:277-81.

28. Schoneberger $\boldsymbol{D}$. Influence of the immunostimulating effects of the pressed juice of herbal Echinacea purpureae on the duration and intensity of the common cold. Results of a double-blind clinical trial. Forum Immunol 1992;2:18-22.

29. Barrett B, Vohmann M, Calabrese C. Echinacea for upper respiratory infection. J Fam Pract 1999;48(8):628-35.

30. Melchart D, Walther E, Linde K, Brandmaier R, Lersch C. Echinacea root extracts for the prevention of upper respiratory tract infections: a double-blind, placebo-controlled randomized trial. Arch Fam Med 1998;7:541-5.

31. Gunning K , Steele $\mathbf{P}$. Echinacea for the prevention of upper respiratory tract infections. J Fam Pract 1999;48(2):93.

32. Hoheisel $\mathbf{0}$, Sandberg M, Bertram S, Bulitta M, Schafer M. Echinacea treatment shortens the course of the common cold: a double-blind, placebo-controlled clinical trial. Eur J Clin Res 1997;9:261-9.

33. Brown D. Echinacea shortens the course of the common cold. Q Rev Nat Med 1998:3-4.

34. Dorn M, Knick E, Lewith G. Placebo-controlled, double-blind study of Echinacea pallidae radix in upper respiratory tract infections. Complement Ther Med 1997;5:40-2.

35. Grimm W, Muller HH. A randomized controlled trial of the effect of fluid extract of Echinacea purpurea on the frequency and severity of colds and respiratory infections. Am J Med 1999;106:138-43.

36. Brinkeborn RM, Shah DV, Degenring FH. Echinaforce and other echinacea fresh plant preparations in the treatment of the common cold. A randomized, placebo controlled, double-blind clinical trial. Phytomedicine 1999;6(1):1-5.

37. AltMedDex Database. Echinacea monograph. Englewood, CO: Micromedex, Inc., 1999

38. Hobbs C. Echinacea: a literature review. HerbalGram 1994;30:33-48.

39. Pepping J. Echinacea. Am J Health-Syst Pharm 1999;56(2): 121-2.

40. Bove M. The echinacea species: a research review. $\mathrm{Br} J$ Phytother 1990;1(1):24-7.

41. Jellin JM, Batz F, Hitchens K. Pharmacist's letter/prescriber's letter natural medicines comprehensive database. Stockton, CA: Therapeutic Research Faculty, 1999:304-6. 